\title{
Metodología flipped classroom: percepción de los alumnos de diferentes grados universitarios
}

\section{Flipped classroom methodology: perception of students of different university degrees}

\author{
Cristina Mendaña-Cuervo ${ }^{1}$, Raquel Poy-Castro ${ }^{2}$, Enrique López-González ${ }^{3}$ \\ ${ }^{1}$ Departamento Dirección y Economía de la Empresa, Universidad de León, España (cristina.mendana@unileon.es) \\ ${ }^{2}$ Departamento Didáctica General, Específicas y Teoría de la Educación, Universidad de León, España \\ (rpoyc@unileon.es) \\ ${ }^{3}$ Departamento Dirección y Economía de la Empresa, Universidad de León, España (enrique.lopez@unileon.es)
}

\section{RESUMEN:}

Este trabajo aporta evidencia sobre la percepción de estudiantes de diferentes grados acerca de la metodología flipped classroom en la mejora de aspectos actitudinales y motivacionales y de las competencias adquiridas con su puesta en práctica. La opinión de los alumnos (antes y después de la aplicación) ha sido recogida en un cuestionario ad hoc. La consideración de alumnos de distintos contextos pretende poder generalizar los resultados, dada la interdisciplinariedad de la muestra.

Los resultados avalan positivamente la experiencia, especialmente en la percepción sobre las competencias adquiridas, si bien en relación a las cuestiones actitudinales y motivacionales no resultan concluyentes.

PALABRAS CLAVE: AULA INVERTIDA, PERCEPCIÓN, EXPECTATIVAS, CAMBIO DE ACTITUD, MOTIVACIÓN, COMPETENCIAS.

\footnotetext{
ABSTRACT:

This study provides evidence on the perception of students belonging to different Degrees about the relationship between the Flipped Classroom methodology and the improvement of attitudinal and motivational aspects, and the skills acquired with its implementation. The opinion of the students (before
}

and after the implementation of the methodology) has been collected using an ad hoc questionnaire. Responses from students in different contexts have been considered in order to be able to generalize the results, given the interdisciplinarity of the sample. The results positively endorse the experience, especially in the perception of the competences acquired. However, in relation to the attitudinal and motivational aspects, they are not conclusive.

KEYWORDS: $\quad$ FLIPPED CLASSROOM,
PERCEPTION, EXPECTATIONS, ATTITUDE
CHANGE, MOTIVATION, SKILLS.

\section{INTRODUCCIÓN}

La implantación del Espacio Europeo de Educación Superior (EEES) y sobre todo la generalización del uso educativo de las tecnologías de la información y las comunicaciones, ha conllevado la necesidad de un cambio en la labor docente tradicional para adecuar las metodologías docentes en base a las posibilidades que ofrece el entorno digital, para enseñar de forma distinta, adaptándose a las formas de aprender de los nativos digitales y millennials. De esta forma, han surgido metodologías activas y colaborativas, en las que el profesor actúa más como 
guía, facilitador, mediador o curator del estudiante, ejerciendo un rol de acompañamiento y moderación propiciatoria en el proceso de aprendizaje, donde el estudiante se erige en verdadero protagonista, como propugna el EEES (Bowden, Marton, y Ramírez Moguel, 2012; Pérez de Albéniz Iturriaga, Escolano Pérez, Pascual Sufrate, Lucas Molina y Sastre i Riba, 2015). Así, la docencia universitaria se efectúa en función del aprendizaje de los alumnos, quienes tras la activación de un conjunto de competencias propiciadas por el diseño de la práctica educativa que efectúa el profesor, son capaces de aprender de forma autónoma.

De ahí que no son pocos los docentes universitarios que han implementado nuevas metodologías educativas, en consonancia con los mencionados principios del Plan Bolonia (Rué Domingo, 2007) y avalados por la generalidad en el uso de las nuevas tecnologías que han llegado a las aulas de forma masiva (Holgado Sáez, 2011). Sin embargo, en la mayoría de los casos no se cuestiona la repercusión en las expectativas de los alumnos de dichas metodologías, ni a priori ni una vez puestas en práctica, y aunque son varios los trabajos que ponen de manifiesto que las expectativas positivas hacia una tarea influyen tanto en el proceso de aprendizaje como en los resultados obtenidos (Cerda, Romera, Casas, Pérez y Ortega Ruiz, 2017; Pekrun, 1992; Suárez Riveiro y Fernández Suárez, 2005), no abundan las evidencias de tal circunstancia.

Así, en muchos casos, la valoración de la aplicación de nuevos métodos de trabajo se enfoca básicamente en las calificaciones obtenidas para valorar el resultado de la experiencia (GarcíaMerino, Urionabarrenetxea y Bañales-Mallo, 2016; Albalawi, 2018; Opazo Faundez, Acuña Bastias y Rojas Polanco, 2016). Y, sin embargo, las calificaciones están influenciadas por otros muchos factores que difícilmente pueden tenerse en consideración en su totalidad. En otros casos, el interés se centra en el análisis de determinadas competencias, como la Competencia Digital Docente (Sosa Díaz y Palau Martín, 2018).

Por este motivo, se suscita el interés por conocer las expectativas que genera en los alumnos la posibilidad de implementar una metodología activa (Prieto, Díaz y Santiago, 2014), así como la comparación con las opiniones de los alumnos una vez que ha sido aplicada, con la finalidad de conocer (más allá de los resultados numéricos de las calificaciones) las sensaciones que han suscitado en los alumnos como protagonistas. En este sentido, Butt (2014) pone de manifiesto la importancia de evaluar la percepción de los estudiantes ante esta nueva metodología.

Como es sabido, entre las labores del docente se encuentra diseñar la práctica educativa para facilitar el enlace entre las actividades formativas fuera del aula y aquellas que se producen en la propia aula, con el fin de optimizar la comprensión de los estudiantes en las diferentes actividades (Medina Moya y Jarauta Borrasca, 2013), donde el elemento central es el desarrollo de estrategias colaborativas en el aula, que promuevan la discusión y la reflexión de la instrucción efectuada (Bergmann y Sams, 2013). Entre las diferentes metodologías que potencian un aprendizaje significativo, se encuentra la denominada aula invertida o flipped classroom (FC) (Bergmann y Sams, 2012), cuya utilización está emergiendo en diferentes niveles educativos, si bien aún son escasas las experiencias en el ámbito universitario.

De acuerdo con lo anterior, este trabajo reporta la experiencia del empleo de la metodología del aula invertida, aplicándola en diferentes asignaturas de distintos grados, al objeto de facilitar la comparación entre alumnos de diferentes itinerarios curriculares. De esta forma, la interdisciplinariedad de la muestra puede contribuir a la generalización de los resultados obtenidos.

En concreto, la justificación contextual viene motivada porque, con carácter general, en los últimos cursos en todas las asignaturas implicadas en el proyecto (Tabla 1) se ha venido observando un incremento en el número de alumnos que, o bien abandonan la materia, o bien tienen dificultades para superar el proceso de evaluación continua.

Tabla 1. Asignaturas implicadas en el estudio

\begin{tabular}{|c|c|c|c|c|}
\hline & Asignatura & $\begin{array}{c}\text { Área de } \\
\text { conocimiento }\end{array}$ & Carácter & $\begin{array}{c}\text { Titulación } \\
\text { (Grado) }\end{array}$ \\
\hline A1 & $\begin{array}{c}\text { Contabilidad } \\
\text { de Costes }\end{array}$ & $\begin{array}{c}\text { Economía } \\
\text { Financiera y } \\
\text { Contabilidad }\end{array}$ & $\begin{array}{l}\text { obligatoria } \\
3^{\text {er }} \text { curso }\end{array}$ & $\mathrm{ADE}$ \\
\hline A2 & $\begin{array}{c}\text { Marco } \\
\text { Jurídico } \\
\text { Profesional } \\
\text { de la } \\
\text { Actividad } \\
\text { Física }\end{array}$ & $\begin{array}{l}\text { Educación } \\
\text { Física y } \\
\text { Deportiva }\end{array}$ & $\begin{array}{c}\text { optativa } \\
3^{\mathrm{er}}-4^{\mathrm{o}} \\
\text { cursos }\end{array}$ & $\begin{array}{l}\text { Ciencias } \\
\text { de la } \\
\text { Actividad } \\
\text { Física y el } \\
\text { Deporte }\end{array}$ \\
\hline A3 & Matemáticas & $\begin{array}{l}\text { Matemática } \\
\text { Aplicada }\end{array}$ & $\begin{array}{l}\text { obligatoria } \\
1^{\text {er }} \text { curso }\end{array}$ & Biología \\
\hline
\end{tabular}

De ahí que desde el curso académico 2016/2017 se implementó la metodología activa FC, buscando una mayor implicación de los alumnos en el seguimiento de las asignaturas, asumiendo que un mayor compromiso por su parte pudiera redundar en un aumento en el seguimiento de las asignaturas, 
esto es, podría suponer una mayor motivación para los alumnos y, por ende, mejores resultados académicos. Las conclusiones de esta experiencia particular puede consultarse en Mendaña-Cuervo, Poy-Castro y López-González (2018).

Adicionalmente, más allá del estudio del impacto en términos de resultados (motivación y rendimiento académico), se suscita el interés por conocer la opinión de los alumnos frente a la metodología FC, tanto a priori (expectativas iniciales cuando únicamente se les expuso el funcionamiento, objetivos y propósito de la metodología) como una vez llevada a cabo la experiencia, para contrastar si dichas expectativas iniciales, con independencia de los resultados académicos que origine, se ven corroboradas con su opinión una vez aplicada la misma.

Por tanto, este trabajo detalla el proceso de construcción de un instrumento (cuestionario) que, respetando los requerimientos psicométricos correspondientes, sirva para evaluar las expectativas iniciales de los alumnos ante la aplicación del FC, y una vez llevada a cabo la experiencia práctica, permita recabar nuevamente la opinión de los mismos estudiantes. De esta forma, se podría contrastar si dichas expectativas iniciales se han visto corroboradas con la experiencia práctica.

\section{OBJETIVO}

Con el propósito de valorar la percepción de los estudiantes acerca de la pertinencia del empleo de la nueva metodología, el principal objetivo de este trabajo radica en construir un cuestionario ad hoc, denominado "Percepción de la metodología FC", sometiendo a análisis las propiedades psicométricas del mismo, que permita recabar la información acerca de las expectativas de los estudiantes frente al FC.

En concreto, la validez de contenido y la fiabilidad del mismo fueron las propiedades analizadas para comprobar que el instrumento mide aquello que pretende medir y que la estabilidad temporal de las puntuaciones obtenidas es adecuada.

Validado el instrumento, se someterá a la consideración de los mismos alumnos antes de la experiencia (expectativas iniciales) y una vez realizada la experiencia, lo que permitirá obtener evidencia sobre su percepción del funcionamiento real de esta metodología.

El hecho de disponer de las percepciones iniciales (expectativas) y la valoración final (opinión tras la experiencia) permitirá a su vez comparar esa información y conocer si la implementación práctica del aula invertida (FC), en opinión de los alumnos, puede considerarse como una experiencia positiva o si, por el contrario, las expectativas generadas no se han visto corroboradas con la puesta en práctica de la metodología.

Además, el hecho de aplicarlo a asignaturas dispares, permitirá comparar los resultados obtenidos de las opiniones de alumnos de diferentes ramas de conocimiento, de distintos cursos de grado y con asignaturas de carácter diverso (obligatorias y optativas), ya que la mayoría de las experiencias se centran en una única materia o contenido (Froehlich, 2018; Salas-Rueda y Lugo-García, 2012).

\section{DISEÑO Y DESARROLLO}

\subsection{Diseño del cuestionario}

En esta primera parte se procede a describir el proceso de elaboración y validación del instrumento a utilizar para la medición de la percepción de los alumnos ante la metodología FC.

\subsubsection{Redacción de los ítems}

La redacción inicial de los ítems fue llevada a cabo por especialistas en la materia, diferenciando dos bloques de contenido sobre los que indagar: por un lado, aspectos actitudinales y motivacionales (frente a otras metodologías tradicionales) y, por otro lado, el desarrollo de competencias genéricas que la metodología FC potencia de forma prioritaria. En el anexo I se recoge la redacción de los ítems que constituyen la versión inicial del cuestionario, previa a la disposición por parte de los alumnos de la versión final (anexo II).

En aras al cumplimiento del proceso de validación, dicha versión inicial fue sometida a validación por un grupo de expertos, cuyos criterios utilizados se concretan más adelante.

De forma complementaria a la redacción de los ítems, se elaboraron las instrucciones para clarificar las tareas que los jueces deberían llevar a cabo. En concreto, para cada uno de los 18 ítems debían juzgar tres parámetros (pertinencia, relevancia y grado de claridad del ítem), teniendo que puntuar cada ítem y parámetro en una escala tipo Likert de 1 a 4 (siendo 1 "En desacuerdo", 2 "Parcialmente de acuerdo", 3 "Bastante de acuerdo" y 4 "Totalmente de acuerdo").

A este respecto, la "Pertinencia" pretende valorar si el ítem pertenece a la dimensión en la que se incluye; la "Relevancia" alude a la importancia de cada ítem, mientras que la "Claridad" se refiere a si el ítem se entiende sin dificultad. 
Finalmente, los jueces podían realizar las observaciones que considerasen oportunas en el espacio habilitado al efecto, así como sugerir modificaciones en la redacción de los ítems e incluso proponer la eliminación de alguno, previa justificación de su propuesta. Al término de su evaluación tenían la opción de valorar con una puntuación global numérica (de 0 a 10) la adecuación del cuestionario al propósito del mismo, además de efectuar observaciones de mejora.

\subsubsection{Selección de expertos}

Como criterio de selección de los expertos, siguiendo a Skjong y Wentworth (2000), se optó por la selección de los mismos en cumplimiento de los siguientes requisitos: dilatada trayectoria académica e investigadora, principalmente en el campo de la educación, y reconocimiento en la comunidad científica como expertos cualificados a los propósitos que se pretenden.

La modalidad de evaluación fue individual, a través de la plantilla escrita elaborada al efecto y con las instrucciones mencionadas, sin mantener contacto entre ellos.

\subsubsection{Validación del cuestionario}

A los efectos de legitimar el cuestionario se llevó a cabo un estudio de validez de contenido, así como de la fiabilidad del mismo.

\subsubsection{Validez de contenido}

La validez de contenido del cuestionario se fundamentó en el juicio de expertos, complementando esta información de carácter cualitativo con un análisis cuantitativo acerca de los indicadores estadísticos de tendencia central (media y desviación típica, principalmente), con el fin de contrastar las opiniones de los jueces.

El criterio utilizado para incorporar un ítem a la versión final del instrumento ha sido la existencia de un acuerdo de al menos el $80 \%$ de los jueces en los parámetros de pertinencia y relevancia (Hyrkäs, Appelqvist-Schmidlechner y Oksa, 2003). A su vez, la claridad ha sido evaluada desde una perspectiva descriptiva apoyada en los estadísticos mencionados.

En base a las respuestas obtenidas de los jueces y a las observaciones aportadas se procedió a la validación en los siguientes aspectos:

\section{A.1. Parámetro "Claridad"}

Los resultados obtenidos en la valoración del parámetro "Claridad" afecta a los ítems 1, 2, 5, 8 y 16 , dando el resto de los ítems que componen el cuestionario por válidos en base a los resultados obtenidos para este parámetro.

Las modificaciones efectuadas se recogen a modo de resumen en la Tabla 1, en la que se muestran los ítems antes y después de someter el cuestionario al proceso de validación.

Tabla 1. Modificaciones en los ítems tras las valoraciones de los jueces

\begin{tabular}{|c|c|}
\hline Versión inicial (anexo I) & Versión final (anexo II) \\
\hline $\begin{array}{l}\text { Ítem } 1 . \text { Me parece } \\
\text { pertinente para trabajar los } \\
\text { contenidos de esta } \\
\text { asignatura }\end{array}$ & $\begin{array}{l}\text { Esta metodología me parece } \\
\text { adecuada para trabajar los } \\
\text { contenidos de esta } \\
\text { asignatura }\end{array}$ \\
\hline $\begin{array}{l}\text { Ítem } 2 . \text { Mis calificaciones } \\
\text { con esta metodología van } \\
\text { a mejorar }\end{array}$ & $\begin{array}{l}\text { Mis conocimientos van a } \\
\text { mejorar con esta } \\
\text { metodología }\end{array}$ \\
\hline \multirow[t]{2}{*}{$\begin{array}{l}\text { Ítem } 5 \text {. Voy a emplear más } \\
\text { tiempo y esfuerzo que con } \\
\text { la metodología tradicional }\end{array}$} & $\begin{array}{l}\text { Voy a emplear más tiempo } \\
\text { que con la metodología } \\
\text { tradicional }\end{array}$ \\
\hline & $\begin{array}{l}\text { Voy a necesitar esforzarme } \\
\text { más que con la metodología } \\
\text { tradicional }\end{array}$ \\
\hline $\begin{array}{l}\text { Ítem } 8 . \mathrm{Mi} \text { aprendizaje } \\
\text { será más experimental o } \\
\text { práctico }\end{array}$ & Eliminado \\
\hline $\begin{array}{l}\text { Ítem } 16 . \text { Mejoraré mi } \\
\text { capacidad de pensamiento } \\
\text { crítico, abstracto, de } \\
\text { análisis y síntesis }\end{array}$ & $\begin{array}{l}\text { Mejoraré mi capacidad de } \\
\text { analizar, sintetizar y extraer } \\
\text { conclusiones (pensamiento } \\
\text { crítico) }\end{array}$ \\
\hline
\end{tabular}

Concretamente, los ítems afectados por las propuestas de los expertos han sido:

Ítem 1. Tras las observaciones efectuadas por dos jueces que afirman que la palabra "pertinente" puede inducir a error, y dado que la media acerca de la "Claridad" es próxima a 3 (en concreto 3,2 ) y la desviación típica es 1,25 se procede a la modificación, dada la variabilidad observada (Gráfico 1).

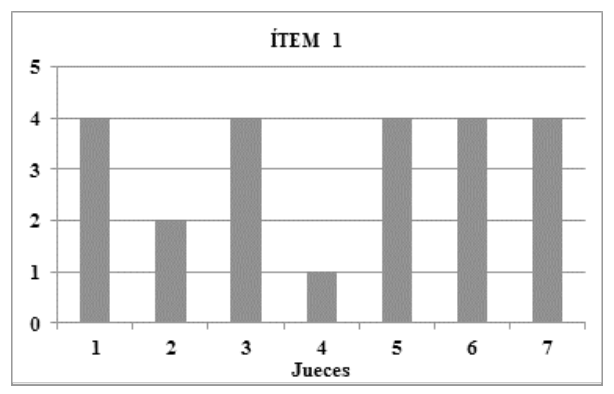

Gráfico 1. Valoraciones jueces ítem 1

Ítem 2. En este ítem también se modificó la redacción (Tabla 1), motivada por las observaciones de los expertos (Gráfico 2) que indican que el término "calificaciones" alude a un resultado final 
cuantitativo que traduce en términos numéricos el grado de adquisición de los conocimientos. Sin embargo, lo que se pretende es que el estudiante analice si la nueva metodología le permite adquirir de forma significativa los conocimientos y no tanto la traslación de estos en calificaciones.

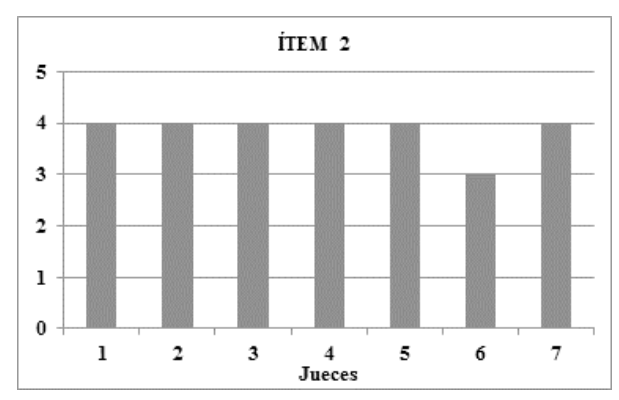

Gráfico 2. Valoraciones jueces ítem 2

Ítem 5. En este caso se reformuló el ítem porque medía dos aspectos diferentes: por un lado, el tiempo $\mathrm{y}$, por otro, el esfuerzo, por lo que se dividió en dos (Tabla 1). El Gráfico 3 muestra la dispersión en las respuestas de los jueces, avalando esta opción.

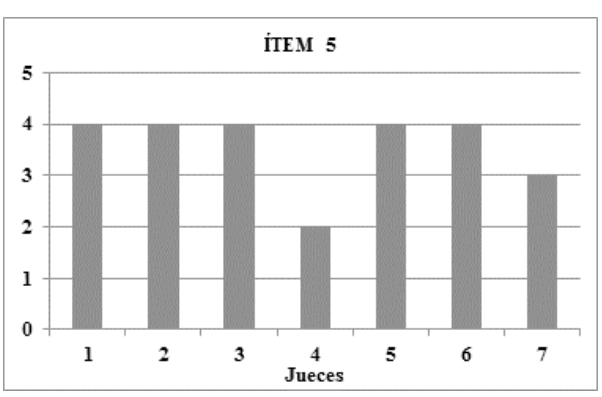

Gráfico 3. Valoraciones jueces ítem 5

Ítem 8. La decisión de eliminar el ítem (Tabla 1) está basada en la redundancia con el contenido de los ítems 9 y 10, manifestado por las opiniones de los jueces que afirman que los contenidos de por sí están más relacionados con un enfoque práctico. Este hecho queda constatado con la elevada desviación típica $(1,13)$ como se visualiza en el Gráfico 4.

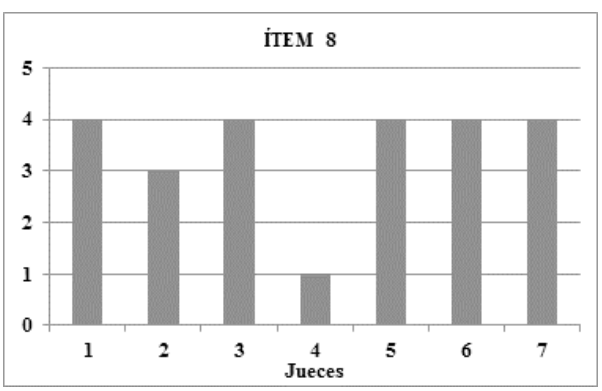

Gráfico 4. Valoraciones jueces ítem 8

Ítem 16. Este ítem se redactó de nuevo (Tabla 1) para explicitar en qué consiste la capacidad de pensamiento crítico, integrando tres aspectos: análisis, síntesis y conclusiones. Nuevamente, la elevada desviación típica $(1,13)$ evidencia esta necesidad, que recoge también el Gráfico 5.

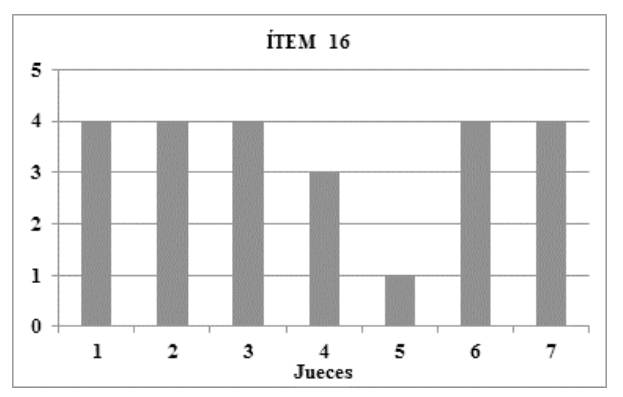

Gráfico 5. Valoraciones jueces ítem 16

\section{A.2. Parámetros "Pertinencia" y "Relevancia"}

A tenor de la evidencia obtenida en relación a estos parámetros, el único ítem que pudiera requerir un análisis en profundidad es el ítem 10 (Tabla 2) por mostrar una desviación típica superior a 1 en ambos casos, aunque presenta una media superior a 3 .

Tabla 2 .Valores estadísticos de los parámetros "Pertinencia" y "Relevancia"

\begin{tabular}{ccccc}
\hline & \multicolumn{2}{c}{ Pertinencia } & \multicolumn{2}{c}{ Relevancia } \\
Ítem & Media & D. típica & Media & D. típica \\
\hline 1 & 3,86 & 0,38 & 3,86 & 0,38 \\
2 & 3,86 & 0,38 & 3,86 & 0,38 \\
3 & 3,86 & 0,38 & 3,86 & 0,38 \\
4 & 3,86 & 0,38 & 3,86 & 0,38 \\
5 & 3,57 & 0,79 & 3,86 & 0,38 \\
6 & 4,00 & 0,00 & 3,86 & 0,38 \\
7 & 3,86 & 0,38 & 3,71 & 0,49 \\
8 & 3,86 & 0,38 & 4,00 & 0,00 \\
9 & 3,71 & 0,49 & 3,86 & 0,38 \\
10 & 3,14 & 1,21 & 3,14 & 1,21 \\
11 & 4,00 & 0,00 & 4,00 & 0,00 \\
12 & 3,71 & 0,76 & 3,86 & 0,38 \\
13 & 4,00 & 0,00 & 4,00 & 0,00 \\
14 & 4,00 & 0,00 & 3,71 & 0,76 \\
15 & 3,71 & 0,49 & 3,86 & 0,38 \\
16 & 3,86 & 0,38 & 3,71 & 0,76 \\
17 & 3,86 & 0,38 & 3,57 & 0,79 \\
18 & 3,86 & 0,38 & 3,71 & 0,49 \\
\hline
\end{tabular}

La posible explicación de la desviación observada se fundamenta en la ya comentada reiteración del contenido de este ítem con el ítem 8 que, como se describió en el apartado anterior, ha sido eliminado por este motivo.

Asimismo, como se puede observar en los gráficos 6 y 7 los promedios obtenidos muestran semejanzas altas, indicativo de la claridad y estabilidad de los enunciados. 


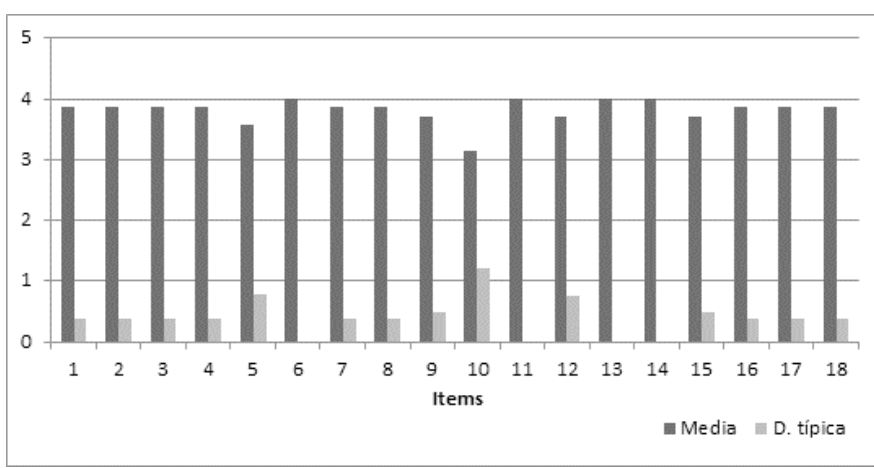

Gráfico 6. Estadísticos parámetro "Pertinencia"

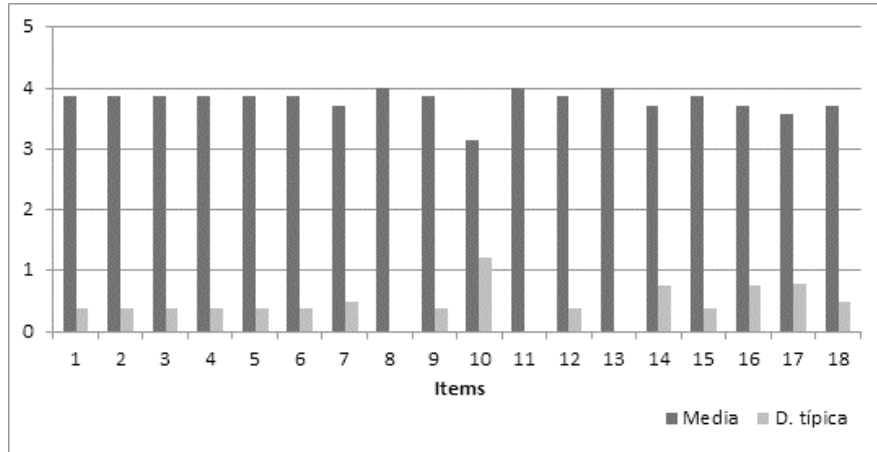

Gráfico 7. Estadísticos parámetro "Relevancia”

\subsubsection{Fiabilidad del instrumento}

Para validar el instrumento, entre los diversos procedimientos existentes para calcular la confiabilidad del mismo, se optó por el método de consistencia interna alfa de Cronbach, por presentar la ventaja de no ser necesario dividir en dos mitades a los ítems del instrumento, sino que simplemente puede aplicarse la medición y calcular el coeficiente.

En relación a este indicador, no existe consenso sobre el valor que debe arrojar el coeficiente, sino que son varios los criterios que diferentes autores han considerado como valor mínimo aceptable (Celina Oviedo y Campo-Arias, 2005; Hernández Sampieri, Fernández Collado, y Baptista Lucio, 2010).

En este caso concreto, dado que los ítems se valoran en la misma unidad de medida, cuanto mayor sea el alfa de Cronbach mayor será la correlación entre los ítems $\mathrm{y}$, por tanto, más probable será que el instrumento sea consistente. En este trabajo se siguen las recomendaciones de George y Mallery (2003, p. 231) que estipulan que valores por encima 0,9 es excelente y por encima de 0,8 ya se puede considerar bueno.

En primer término, se calculó el coeficiente de alfa de Cronbach en una muestra piloto y para confirmar su valor se calculó en la muestra final de las diferentes asignaturas en las que se efectuó la implantación de la metodología $F C$.

De acuerdo con las consideraciones efectuadas en el apartado 3, el cuestionario validado se administró a un grupo piloto de 20 alumnos tomados al azar, dando como resultado un alfa de Cronbach de 0,837 (Tabla 3).

Tabla 3. Estadístico de fiabilidad del cuestionario piloto

\section{Estadísticas de fiabilidad}

\begin{tabular}{c|c} 
Alfa de Cronbach & N de elementos \\
\hline, 837 & 20 \\
\hline
\end{tabular}

Finalmente, una vez contrastada la fiabilidad del instrumento, se procedió a su cálculo en la muestra final de tres asignaturas impartidas en el primer cuatrimestre del curso 2016/2017 de diferentes títulos de grado (Tabla 4), lo que permitió confirmar la confiabilidad del mismo con carácter general al obtener en todos los casos valores superiores a 0,8 .

Tabla 4. Estadístico de fiabilidad del cuestionario final en la muestra de referencia de las diferentes asignaturas

\begin{tabular}{c|c} 
& $\begin{array}{c}\text { Estadísticas de fiabilidad } \\
\text { Alfa de Cronbach }\end{array}$ \\
\hline Asignatura &, 893 \\
\hline A2 &, 873 \\
\hline A3 &, 844 \\
\hline
\end{tabular}

En consecuencia, analizadas las características psicométricas del cuestionario y en base a los resultados obtenidos, se puede concluir que el instrumento puede utilizarse para medir las expectativas por parte de los estudiantes acerca de la implementación de la metodología FC. De ahí que dicho cuestionario haya sido utilizado en el siguiente aspecto de la investigación.

\subsection{Desarrollo de la investigación}

El cuestionario validado, y una vez comprobada su fiabilidad, se aplicó a los alumnos antes y después de la experiencia, de forma que con dicha información se planteó la siguiente hipótesis a contrastar:

$\mathrm{H}_{0}$ : Las expectativas de los alumnos son las mismas que tras la experiencia

$\mathrm{H}_{1}$ : Las expectativas de los alumnos no son las mismas que tras la experiencia

Además, como se ha comentado, en el cuestionario se consideraron dos grupos de ítems relativos a dos cuestiones básicas (aspectos actitudinales y motivacionales, y desarrollo de competencias genéricas). En concreto, los ítems 
fueron agrupados en relación a cada una de las dos cuestiones anteriores, como se refleja en la tabla 5. De ahí que el análisis se haya realizado a dos niveles: uno, para todos los ítems del cuestionario y, dos, para cada uno de los dos grupos de ítems. En ambos casos para cada una de las tres asignaturas.

Tabla 5. Grupos de ítems

\begin{tabular}{ll}
\hline Aspectos a considerar & Ítems \\
\hline G1. Cuestiones actitudinales & $1-3-4-5-6-7$ \\
y motivacionales & $2-8-9-10-11-12-13-14-15-16-$ \\
G2. Competencias adquiridas & $17-18$ \\
\hline
\end{tabular}

Por su parte, el contraste de hipótesis deberá ser realizado para cada uno de los grupos de alumnos de cada una de las asignaturas implicadas en el estudio, de forma que se pueda contrastar en cada asignatura si mejoran las expectativas con la experiencia o por el contrario empeoran, lo que supondría que la nueva metodología no ha supuesto la mejora en la percepción que tienen los alumnos sobre la nueva metodología. Además, el hecho de obtener información de diferentes muestras de alumnos, en función del resultado, podrá corroborar o no los datos parciales de cada asignatura.

Dado que se trata del mismo grupo evaluado en dos ocasiones diferentes, se plantea aplicar la prueba $t$ de muestras dependientes o apareadas (una prueba $t$ de mediciones repetitivas), si bien con anterioridad será preciso someter los datos a las pruebas estadísticas correspondientes con el fin de comprobar los supuestos de normalidad necesarios para la aplicación de una técnica paramétrica. En caso contrario, se optaría por la prueba de los rangos de Wilcoxon.

\subsubsection{Población y muestra}

La población está compuesta en todos los casos por estudiantes de la Universidad de XXX, siendo la muestra un grupo de alumnos de cada una de las asignaturas recogidas en la Tabla 1, con los valores que se reflejan en la tabla 6 .

Tabla 6. Población y muestra

\begin{tabular}{cc}
\hline Asignatura & Número de alumnos \\
\hline A1 & 31 \\
A2 & 18 \\
A3 & 25 \\
\hline
\end{tabular}

A este respecto, comentar que la implementación práctica de la experiencia se llevó a cabo solamente con un grupo de alumnos (grupo experimental, GE), ya que a fin de poder evaluar comparativamente resultados en relación a la motivación y al rendimiento académico de los alumnos (Mendaña Cuervo, Poy Castro, González Fernández, Arana Suárez y López González, 2017), se mantuvo un grupo con la metodología tradicional (grupo control, GC). De ahí que el estudio realizado se haya llevado a cabo solamente con los datos recopilados de los alumnos del grupo experimental, que son los que efectivamente han sido sometidos a la nueva metodología.

\subsubsection{Recolección y procesamiento de datos}

La recolección de datos se ha realizado implementado el cuestionario mencionado (antes y después de la experiencia) en la plataforma Moodle, en la web de cada asignatura.

Las cuestiones recogidas en el mismo son idénticas al cuestionario inicial (anexo II), a excepción de la percepción temporal (futuro/pasado) en las preguntas ${ }^{1}$. Además, en el cuestionario posterior se ha añadido un ítem ${ }^{2}$ a fin de conocer la opinión de los alumnos en relación a la posibilidad de implementar la metodología en el resto de la asignatura. Los datos obtenidos, descargados de Moodle, han sido procesados en SPSS.

\section{RESULTADOS Y DISCUSIÓN}

En todos los casos se dispone de la información sobre las respuestas del grupo de alumnos sometido a la metodología, antes y después, de forma que para proceder al contraste de medias se calculan las medias para cada ítem. Los resultados obtenidos en cada uno de los dos planteamientos mencionados en el apartado "Diseño de la investigación" han sido los que se describen a continuación.

\subsection{Resultados a nivel global}

En relación a las respuestas totales obtenidas, con la finalidad de comprobar si es factible la aplicación de una técnica paramétrica para el contraste, se han sometido las respuestas de los grupos de cada asignatura (antes $-\mathrm{A}-\mathrm{y}$ después $-\mathrm{D}-$ de la experiencia) a las pruebas de normalidad (tabla 7).

Por tanto, es factible la aplicación de una técnica paramétrica en todas las asignaturas y, en este caso, se optó por la prueba t, para someter a contraste la hipótesis:

\footnotetext{
En la versión final del cuestionario, una vez llevada a cabo la experiencia, simplemente se han expresado las mismas cuestiones realizando las preguntas en tiempo pasado, razón por la cual no se ha reiterado su inclusión en este trabajo.

Ítem nuevo. "Me gustaría mantener esta metodología frente a la metodología tradicional en el resto del curso".
} 
$\mathrm{H}_{0}$ : La media de las poblaciones son iguales

$\mathrm{H}_{1}$ : La media de las poblaciones no son iguales

Tabla 7. Pruebas de normalidad para cada asignatura

\begin{tabular}{|lc|c|c|c|c|c|}
\hline & \multicolumn{3}{c}{ Kolmogorov-Smirnov $^{\mathrm{a}}$} & \multicolumn{3}{c|}{ Shapiro-Wilk } \\
& Estadístico & $\mathrm{gl}$ & Sig. & Estadístico & gl & Sig. \\
\hline A1_Antes &, 184 & 18 &, 110 &, 934 & 18 &, 231 \\
\hline A1_Después &, 100 & 18 &, $200^{*}$ &, 973 & 18 &, 852 \\
\hline A2_Antes &, 129 & 18 &, $200^{*}$ &, 956 & 18 &, 534 \\
\hline A2_Después &, 192 & 18 &, 078 &, 941 & 18 &, 297 \\
\hline A3_Antes &, 130 & 18 &, $200^{*}$ &, 969 & 18 &, 785 \\
\hline A3_Despues &, 195 & 18 &, 068 &, 954 & 18 &, 491 \\
\hline *. Esto es un límite inferior de la significación verdadera. \\
a. Corrección de significación de Lilliefors \\
\hline
\end{tabular}

Los resultados obtenidos en la prueba $t$ para cada asignatura se recogen en la Tabla 8 , en la que se puede observar que el resultado obtenido en las tres asignaturas supone rechazar la hipótesis nula, lo que implica aceptar que las medias de las poblaciones antes y después de la experiencia no son iguales. Dado que la media ha aumentado en todos los casos, eso supone que las expectativas de los alumnos sobre la metodología FC han mejorado con la experiencia.

Tabla 8. Prueba $t$ para cada asignatura (Antes-Después)

\begin{tabular}{|c|c|c|c|c|c|c|c|c|}
\hline & & Diferer & cias empa & arejadas & & & & \\
\hline & & & Media & $\begin{array}{l}95 \% \text { de } \\
\text { de conf } \\
\text { la dif }\end{array}$ & $\begin{array}{l}\text { ntervalo } \\
\text { anza de } \\
\text { rencia }\end{array}$ & & & \\
\hline & Media & Est. & estándar & Inf. & Sup. & $\mathrm{t}$ & $\mathrm{gl}$ & (bil.) \\
\hline A1 &,- 4370 & ,4278 & , 1008 &,- 6498 &,- 2242 & $-4,33$ & 17 & ,000 \\
\hline A2 &,- 1882 & ,2319 & ,0546 &,- 3036 &,- 0729 & $-3,44$ & 17 & ,003 \\
\hline A3 &,- 3977 & ,3944 & ,0929 &,- 5939 &,- 2016 & $-4,27$ & 17 & ,001 \\
\hline
\end{tabular}

\subsection{Resultados a nivel de grupos de ítems}

En este caso, el objetivo es conocer los resultados en cada uno de los dos grupos en que se han dividido los ítems del cuestionario (tabla 5), para contrastar las mismas hipótesis del apartado anterior.

Para ello, se realizaron de nuevo las pruebas de normalidad de los datos obtenidos de las respuestas de los alumnos, atendiendo en este caso tanto a la asignatura como a las respuestas obtenidas para cada grupo de ítems (tabla 9).
Tabla 9. Pruebas de normalidad para cada asignatura y para cada grupo de ítems

\begin{tabular}{|c|c|c|c|c|c|c|c|}
\hline & & $\begin{array}{r}\text { Kolmo } \\
\text { Smir }\end{array}$ & hov $^{\mathrm{a}}$ & & Shapiro & $-W i$ & \\
\hline & Grupo & Estadístico & $\mathrm{gl}$ & Sig. & Estadístico & $\mathrm{gl}$ & Sig. \\
\hline & 1 & ,215 & 6 & $200^{*}$ & ,924 & 6 &, 537 \\
\hline & 2 & ,270 & 12 & ,016 & ,890 & 12 & ,118 \\
\hline & 1 & , 170 & 6 &, $200^{*}$ & ,963 & 6 & 841 \\
\hline $\mathrm{A}$ & 2 & , 164 & 12 &, $200^{*}$ & 959 & 12 & ,775 \\
\hline & 1 & ,248 & 6 & $200^{*}$ & ,898 & 6 & ,360 \\
\hline & 2 & , 143 & 12 &, $200^{*}$ & ,920 & 12 & ,284 \\
\hline & 1 & 217 & 6 & $200^{*}$ & ,945 & 6 & ,700 \\
\hline & 2 & ,218 & 12 & , 122 & ,935 & 12 & ,440 \\
\hline & 1 & , 158 & 6 & $200^{*}$ & 959 & 6 & ,813 \\
\hline & 2 & ,131 & 12 &, $200^{*}$ & ,964 & 12 & ,837 \\
\hline & 1 & ,206 & 6 &, $200^{*}$ & 969 & 6 & 888 \\
\hline & 2 & ,275 & 12 & ,012 & ,900 & 12 & , 161 \\
\hline
\end{tabular}

*. Esto es un límite inferior de la significación verdadera.

a. Corrección de significación de Lilliefors

(A) Antes

(B) Después

Como se puede observar, para el Grupo 1 "Cuestiones actitudinales y motivacionales" es factible en todas las asignaturas aplicar una técnica paramétrica, habiendo optado nuevamente -por comparabilidad- por la prueba t. Los datos obtenidos para este grupo en cada asignatura se muestran en la tabla 10.

Tabla 10. Pruebas $t$ para el Grupo 1 "Cuestiones actitudinales y motivacionales" (Antes-Después)

\begin{tabular}{|c|c|c|c|c|c|c|c|c|}
\hline & & Difer & encias emp & parejadas & & & & \\
\hline & & & $\begin{array}{c}\text { Media } \\
\text { error }\end{array}$ & $\begin{array}{r}95 \% \text { de int } \\
\text { confianz } \\
\text { difere }\end{array}$ & $\begin{array}{l}\text { rvalo de } \\
\text { de la } \\
\text { cia }\end{array}$ & & & \\
\hline & Media & Est. & estándar & Inf. & Sup. & $\mathrm{t}$ & gl & (bil.) \\
\hline A1 &,- 59500 & ,37372 & ,15257 &,- 98720 &,- 20280 & $-3,900$ & 5 & ,011 \\
\hline A2 &,- 25926 & ,24762 & , 10109 &,- 51912 &, 00060 & $-2,565$ & 5 &, 050 \\
\hline A3 &,- 29333 & ,31258 & ,12761 &,- 62137 &, 03470 & $-2,299$ & 5 &, 070 \\
\hline
\end{tabular}

Los resultados obtenidos en relación al Grupo 1 suponen rechazar la hipótesis nula en el caso de la asignatura $\mathrm{A} 1$, mientras que en el caso de A3 se acepta dicha hipótesis. La asignatura A2, para el nivel de significación propuesto, se encuentra al límite. 
Nuevamente, dado que la media ha aumentado en todos los casos, permite asumir que solamente en el caso de la asignatura A1 ha habido un incremento significativo en las expectativas de los alumnos en relación a cuestiones actitudinales y motivacionales a través de la aplicación de la metodología FC.

En relación al Grupo 2 "Competencias adquiridas", únicamente en el caso de la asignatura A2 se da la circunstancia de normalidad, aplicando por tanto la prueba t, cuyo resultado se recoge en la tabla 11 .

Tabla 11. Prueba t para el Grupo 2 "Competencias adquiridas”, asignatura A2 (Antes-Después)

\begin{tabular}{|c|c|c|c|c|c|c|c|c|}
\hline & & Diferen & las empa & ejadas & & & & \\
\hline & & & $\begin{array}{l}\text { Media } \\
\text { error }\end{array}$ & $\begin{array}{l}95 \% \text { de } \\
\text { de conf } \\
\text { la dif }\end{array}$ & $\begin{array}{l}\text { ntervalo } \\
\text { anza de } \\
\text { encia }\end{array}$ & & & \\
\hline & Media & estándar & ar & Inf. & Sup. & $\mathrm{t}$ & $\mathrm{gl}$ & (bil.) \\
\hline A2 &,- 15278 & ,22613 & ,06528 &,- 29646 &,- 00910 & & 11 & ,039 \\
\hline & & & & & & 2,340 & & \\
\hline
\end{tabular}

En el caso de las asignaturas A1 y A3, ante la imposibilidad de utilizar la técnica anterior, se aplicó la técnica alternativa a la paramétrica, en concreto la prueba de los rangos de Wilcoxon, ya que la muestra es relacionada, obteniendo los datos de la tabla 12.

Tabla 12. Prueba Wilcoxon para el Grupo 2

"Competencias adquiridas", asignaturas A1 y A3

\begin{tabular}{|lrr|}
\hline \multicolumn{2}{|c|}{ A1: D-A } & \multicolumn{1}{c|}{ A3: D-A } \\
\hline$Z$ & $-3,201^{\mathrm{b}}$ & $-3,161^{\mathrm{b}}$ \\
\hline Sig. asintótica (bilateral) &, 001 &, 002 \\
\hline $\begin{array}{l}\text { a. Prueba de rangos con signo de Wilcoxon } \\
\text { b. Se basa en rangos negativos. }\end{array}$ \\
\hline
\end{tabular}

Los resultados obtenidos en este caso (grupo 2) implica rechazar la hipótesis nula en todas las asignaturas, por lo que en todos los casos los alumnos manifiestan un incremento en las competencias adquiridas con la metodología evaluada.

A mayores, en relación al cuestionario final, como se comentó anteriormente se reflejó un nuevo ítem o pregunta con la siguiente redacción: "Me gustaría mantener esta metodología frente a la metodología tradicional en el resto del curso". Con este proceder se trataba de conocer no solamente su percepción hacia la metodología FC una vez llevada a cabo la experiencia, sino también su predisposición final hacia la misma. Los datos resumidos de las respuestas obtenidas se muestran en la tabla 13.
Tabla 13. Porcentajes relativos de las frecuencias, respuesta ítem 19

\begin{tabular}{|lccc|}
\hline Respuesta/Asignatura & A1 & A2 & A3 \\
\hline Muy poco & $0,00 \%$ & $5,56 \%$ & $4,00 \%$ \\
Poco & $0,00 \%$ & $5,56 \%$ & $4,00 \%$ \\
Suficiente & $3,45 \%$ & $16,67 \%$ & $20,00 \%$ \\
Bastante & $17,24 \%$ & $38,89 \%$ & $48,00 \%$ \\
Mucho & $79,31 \%$ & $33,33 \%$ & $24,00 \%$ \\
\hline
\end{tabular}

En este caso, un simple análisis de frecuencias da como resultado que mayoritariamente están predispuestos con un grado de "Bastante" o "Mucho" a que toda la asignatura se imparta bajo la metodología FC en todas las asignaturas. De hecho, los porcentajes acumulados para estas dos valoraciones ascienden al $96,55 \%$ para $\mathrm{A} 1$, al $72,22 \%$ para A2 y al $72 \%$ para A3.

\section{CONCLUSIONES Y APORTACIONES}

La principal evidencia obtenida de las experiencias realizadas ratifica la predisposición de los alumnos hacia la metodología FC, medida en términos de su opinión sobre la posibilidad de mantenerla como método de trabajo, a tenor de los resultados obtenidos, cabe considerarse como muy positiva en todos los casos.

Como principales contribuciones derivadas de este trabajo cabe comentar, en primer lugar, la elaboración de un instrumento que permite, con carácter general, valorar las expectativas (ante y a posteriori) de los alumnos ante la implementación de la metodología activa FC.

Por otra parte, la aplicación del cuestionario a alumnos de diferentes asignaturas y estudias permite concluir que, en base a las respuestas obtenidas de los alumnos, la experiencia práctica de la metodología flipped classroom ha mejorado las expectativas que los alumnos tenían antes de su puesta en práctica en todos los casos.

En el estudio se han reflejado opiniones de alumnos de diferentes Grados y distintas asignaturas, tanto en relación su carácter (obligatorias y optativas) como al curso en que se imparten.

El resultado puede suponer que, con independencia del tipo de alumnado, la metodología FC puede ser válida desde la perspectiva de los alumnos, en el sentido de que sus expectativas han mejorado con la experiencia. 
En segundo lugar, considerando la posibilidad de un análisis de ítems agrupados en relación con dos aspectos fundamentales como son "Cuestiones actitudinales y motivacionales" y "Competencias adquiridas" los resultados han sido dispares: Así, en el primer caso, si bien la media ha aumentado en todas las asignaturas, solamente en una (A1) dicho incremento ha sido significativo, en otro caso está al límite y en el caso de A3 no ha sido significativo. Esta diferencia puede explicarse debido al perfil del alumnado, ya que probablemente en alumnos de cursos superiores (como es el caso de A1 y A2) un nuevo planteamiento de enseñanza-aprendizaje puede ser motivador y suponer un cambio en su actitud hacia la asignatura, mientras que entendemos que en el caso de alumnos de nuevo ingreso (la asignatura A3 se imparte en el primer curso en el primer semestre) el efecto de una nueva forma de abordar la asignatura no les resulta tan atrayente precisamente por la novedad que supone en sus planteamientos. En el segundo caso, en relación a las competencias, en todos los casos las expectativas de los alumnos han aumentado de forma significativa con la experiencia. Esto nos permite afirmar que, en base a estos resultados, con independencia del perfil del alumnado, la metodología FC puede resultar un elemento facilitador de la aprehensión de competencias.

\section{REFERENCIAS}

Albalawi, A. S. (2018). The Effect of Using Flipped Classroom in Teaching Calculus on Students' Achievements at University of Tabuk. International Journal of Research in Education and Science, 4(1), 198-207.

Bergmann, J. y Sams, A. (2012). Flip Your Classroom: Reach Every Student in Every Class Every Day (Internatio). Washington, DC.

Bergmann, J. y Sams, A. (2013). Flip Your Students' Learning. Educational Leadership, 70(6), 16-20.

Bowden, J. A., Marton, F. y Ramírez Moguel, E. (2012). La Universidad, un espacio para el aprendizaje : más allá de la calidad y la competencia. Madrid: Narcea.

Butt, A. (2014). Student Views on the Use of a Flipped Classroom Approach: Evidence from Australia. Business Education y Accreditation, 6(1), 33-43.

Celina Oviedo, H. y Campo-Arias, A. (2005). Aproximación al uso del coeficiente alfa de Cronbach. Revista Colombiana de Psiquiatría, 34(4), 572-580.

Cerda, G., Romera, E. M., Casas, J. A., Pérez, C. y Ortega Ruiz, R. (2017). Influencia de variables cognitivas y motivacionales en el rendimiento académico en matemáticas en estudiantes chilenos. Educación XX1, 20(2). doi: 10.5944/educxx1.19052

Froehlich, D. E. (2018). Non-Technological Learning Environments in a Technological World: Flipping Comes To The Aid. Journal of New Approaches in Educational Research, 7(2), 88-92. doi: 10.7821/naer.2018.7.304

García-Merino, J. D., Urionabarrenetxea, S. y Bañales-Mallo, A.
(2016). Cambios en metodologías docentes y de evaluación: ¿Mejoran el rendimiento del alumnado universitario? Revista Electrónica de Investigación Educativa, 18(3), 1-19.

George, D. y Mallery, P. (2003). SPSS for Windows step by step: A simple guide and reference. (A. y Bacon., Ed.) $\left(4^{\mathrm{a}}\right)$. Boston.

Hernández Sampieri, R., Fernández Collado, C. y Baptista Lucio, P. (2010). Metodología de la investigación. México, D.F.: McGraw Hill.

Holgado Sáez, C. (2011). Las nuevas tecnologías en los estudios de Derecho en el marco del EEES: Sugerencias didácticas de actividades colaborativas con entornos virtuales. REJIE: Revista Jurídica de Investigación e Innovación Educativa, 3, 93-106.

Hyrkäs, K., Appelqvist-Schmidlechner, K. y Oksa, L. (2003). Validating an instrument for clinical supervision using an expert panel. International Journal of Nursing Studies, 40(6), 619-625. doi: 10.1016/S0020-7489(03)00036-1

Medina Moya, J. L., y Jarauta Borrasca, B. (2013). Enseñanza y aprendizaje en la Educación Superior. Madrid: Síntesis.

Mendaña-Cuervo, C., Poy-Castro, R. y López-González, E. (2018). Análisis comparativo de la puesta en práctica de la metodología Flipped Classroom en los Grados de ADE y Educación Infantil. En A. M. Arnal Pons, S. Barrachina Mir, J. Castelló Benavent, I. Epifanio López, C. Galindo Pastor, P. Gregori Huerta, ... A. L. P. y V. M. García (Eds.), Actas del Congreso Virtual "Avances en Tecnologías, Innovación y Desafios de la Educación Superior» (ATIDES 2018) (pp. 208-220). Universitat Jaume I. doi: 10.6035/InnovacioEducativa.2018.19

Mendaña Cuervo, C., Poy Castro, R. González Fernández, A., Arana Suárez, M. V. y López González, E. (2017). ¿Influye el aula invertida en la motivación y el rendimiento académico de estudiantes universitarios? Revista Infancia, Educación y Aprendizaje, 3(2), 660. doi: 10.22370/ieya.2017.3.2.798

Opazo Faundez, A. R., Acuña Bastias, J. M. y Rojas Polanco, M. P. (2016). Evaluación de metodología Flipped Classroom: primera experiencia. Innoeduca. International Journal of Technology and Educational Innovation, 2(2), 90-99.

doi:

10.24310/INNOEDUCA.2016.V2I2.2030.G1943

Pekrun, R. (1992). The Impact of Emotions on Learning and Achievement: Towards a Theory of Cognitive/Motivational Mediators. Applied Psychology, 41(4), 359-376. doi: 10.1111/j.1464-0597.1992.tb00712.x

Pérez de Albéniz Iturriaga, A., Escolano Pérez, E., Pascual Sufrate, M. T., Lucas Molina, B. y Sastre i Riba, S. (2015). Metacognición en un proceso de aprendizaje autónomo y cooperativo en el aula universitaria. Contextos educativos, (18), 95-108. doi: http://dx.doi.org/10.18172/con.2576

Prieto, A., Díaz, D. y Santiago, R. (2014). Metodologías inductivas. El desafio de enseñar mediante el cuestionamiento y los retos. Barcelona: Editorial Océano S.L.U.

Rué Domingo, J. (2007). Enseñar en la Universidad. El EEES como reto para la Educación Superior. Narcea, S.A. de Ediciones.

Salas-Rueda, R. A. y Lugo-García, J. L. (2012). Impacto del aula invertida durante el proceso educativo superior sobre las derivadas considerando la ciencia de datos y el aprendizaje automático. Edmetic Revista de Educación Mediática y TIC, $8(1), \quad 147-170 . \quad$ doi: 10.21071/edmetic.v8i1.9542 
Skjong, R. y Wentworth, B. (2000). Expert Judgement and risk perception. Recuperado a partir de http://research.dnv.com/skj/Papers/SkjWen.pdf

Sosa Díaz, M. J. y Palau Martín, R. F. (2018). Flipped Classroom para adquirir la Competencia Digital Docente: Una experiencia didáctica en la Educación superior. Píxel-Bit. Revista de Medios y Educación, 0(52), 37-54.

Suárez Riveiro, J. M., y Fernández Suárez, A. P. (2005). Escalas de evaluación de las estrategias motivacionales de los estudiantes, 21, 116-128.

\section{ANEXO I}

\begin{tabular}{|c|c|c|c|}
\hline \multicolumn{4}{|c|}{$\begin{array}{l}\text { Valore de } 1 \text { a } 4 \text { cada uno de los parámetros: } \\
\text { 1. En desacuerdo; } 2 \text {. Parcialmente de acuerdo; } \\
\text { 3. Bastante de acuerdo y } 4 \text {. Totalmente de acuerdo }\end{array}$} \\
\hline & Pertinencia & Relevancia & Claridad \\
\hline \multicolumn{4}{|c|}{ PERCEPCIÓN DE LA METODOLOGÍA FC } \\
\hline $\begin{array}{l}\text { 1. Me parece pertinente para } \\
\text { trabajar los contenidos de esta } \\
\text { asignatura }\end{array}$ & & & \\
\hline $\begin{array}{l}\text { 2. Mis calificaciones con esta } \\
\text { metodología van a mejorar }\end{array}$ & & & \\
\hline $\begin{array}{l}\text { 3. Mi actitud hacia la asignatura } \\
\text { va a mejorar }\end{array}$ & & & \\
\hline $\begin{array}{l}\text { 4. Mi motivación hacia la } \\
\text { asignatura va a aumentar }\end{array}$ & & & \\
\hline $\begin{array}{l}\text { 5. Voy a emplear más tiempo y } \\
\text { esfuerzo que con la } \\
\text { metodología tradicional }\end{array}$ & & & \\
\hline $\begin{array}{l}\text { 6. Mi interacción con el profesor } \\
\text { va a ser más frecuente y } \\
\text { positiva }\end{array}$ & & & \\
\hline $\begin{array}{l}\text { 7. Voy a tener la posibilidad de } \\
\text { trabajar a mi propio ritmo }\end{array}$ & & & \\
\hline $\begin{array}{l}\text { 8. Mi aprendizaje será más } \\
\text { experimental o práctico }\end{array}$ & & & \\
\hline $\begin{array}{l}\text { 9. Me permitirá profundizar más } \\
\text { en los contenidos teóricos }\end{array}$ & & & \\
\hline $\begin{array}{l}\text { 10. Me permitirá profundizar más } \\
\text { en los contenidos prácticos }\end{array}$ & & & \\
\hline $\begin{array}{l}\text { 11. Fomentará mi aprendizaje } \\
\text { autónomo }\end{array}$ & & & \\
\hline $\begin{array}{l}\text { 12. Fomentará mi aprendizaje } \\
\text { colaborativo con los } \\
\text { compañeros }\end{array}$ & & & \\
\hline $\begin{array}{l}\text { 13. Me resultará más fácil aplicar } \\
\text { el conocimiento teórico a } \\
\text { situaciones prácticas }\end{array}$ & & & \\
\hline $\begin{array}{l}\text { 14. Mejoraré mi capacidad de } \\
\text { identificar, plantear y resolver } \\
\text { problemas }\end{array}$ & & & \\
\hline $\begin{array}{l}\text { 15. Mejoraré mi capacidad para } \\
\text { adaptarme a nuevas } \\
\text { situaciones }\end{array}$ & & & \\
\hline $\begin{array}{l}\text { 16. Mejoraré mi capacidad de } \\
\text { pensamiento crítico, abstracto, } \\
\text { de análisis y síntesis }\end{array}$ & & & \\
\hline $\begin{array}{l}\text { 17. Me resultará más fácil } \\
\text { investigar, procesar y analizar } \\
\text { información desde distintas } \\
\text { fuentes }\end{array}$ & & & \\
\hline
\end{tabular}

\begin{tabular}{|l|l|l|l|}
\hline $\begin{array}{l}\text { 18. Podré transmitir con más } \\
\text { facilidad mis ideas, problemas } \\
\text { y soluciones }\end{array}$ & & & \\
\hline
\end{tabular}

\section{ANEXO II}

\begin{tabular}{|c|c|}
\hline $\begin{array}{llll}\text { 1. } & \text { Muy poco } & \text { 2. Poco } & \text { 3.Suficiente } \\
\text { 4. } & \text { Bastante } & \text { 5.Mucho } & \end{array}$ & \\
\hline $\begin{array}{l}\text { 1. Esta metodología me parece adecuada para trabajar los } \\
\text { contenidos de esta asignatura }\end{array}$ & \\
\hline 2. Mis conocimientos van a mejorar con esta metodología & \\
\hline 3. Mi actitud hacia la asignatura va a mejorar & \\
\hline 4. Mi motivación hacia la asignatura va a aumentar & \\
\hline $\begin{array}{l}\text { 5. Voy a emplear más tiempo que con la metodología } \\
\text { tradicional }\end{array}$ & \\
\hline $\begin{array}{l}\text { 6. Voy a necesitar esforzarme más que con la metodología } \\
\text { tradicional }\end{array}$ & \\
\hline $\begin{array}{l}\text { 7. Mi interacción con el profesor va a ser más frecuente y } \\
\text { positiva }\end{array}$ & \\
\hline 8. Voy a tener la posibilidad de trabajar a mi propio ritmo & \\
\hline $\begin{array}{l}\text { 9. Me permitirá profundizar más en los contenidos } \\
\text { teóricos }\end{array}$ & \\
\hline $\begin{array}{l}\text { 10. Me permitirá profundizar más en los contenidos } \\
\text { prácticos }\end{array}$ & \\
\hline 11. Fomentará mi aprendizaje autónomo & \\
\hline $\begin{array}{l}\text { 12. Fomentará mi aprendizaje colaborativo con los } \\
\text { compañeros }\end{array}$ & \\
\hline $\begin{array}{l}\text { 13. Me resultará más fácil aplicar el conocimiento teórico a } \\
\text { situaciones prácticas }\end{array}$ & \\
\hline $\begin{array}{l}\text { 14. Mejoraré mi capacidad de identificar, plantear y } \\
\text { resolver problemas }\end{array}$ & \\
\hline $\begin{array}{l}\text { 15. Mejoraré mi capacidad para adaptarme a nuevas } \\
\text { situaciones }\end{array}$ & \\
\hline $\begin{array}{l}\text { 16. Mejoraré mi capacidad de analizar, sintetizar y extraer } \\
\text { conclusiones (pensamiento crítico) }\end{array}$ & \\
\hline $\begin{array}{l}\text { 17. Me resultará más fácil investigar, procesar y analizar } \\
\text { información desde distintas fuentes }\end{array}$ & \\
\hline $\begin{array}{l}\text { 18. Podré transmitir con más facilidad mis ideas, } \\
\text { problemas y soluciones }\end{array}$ & \\
\hline 19. Género & \\
\hline 20. Edad & \\
\hline $\begin{array}{l}\text { 21. ¿Es el primer año que estás cursando estudios } \\
\text { universitarios? }\end{array}$ & \\
\hline 22. Los enunciados de las preguntas son comprensibles & \\
\hline 23. La extensión del cuestionario te ha parecido adecuada & \\
\hline $\begin{array}{l}\text { 24. Puede añadir cualquier observación en relación a este } \\
\text { cuestionario que considere oportuna }\end{array}$ & \\
\hline
\end{tabular}

